\title{
Propuesta para un registro nacional electrónico de cáncer en Perú, considerando el uso de cuatro importantes fuentes de información electrónica
}

Proposal for a electronic national registry of cancer in Peru, considering the use of four important sources of electronic information

\section{Señor Editor:}

Pow-Sang $\mathrm{M}$ et al (1), menciona que "las tasas de incidencia, según el Registro de Cáncer de Lima Metropolitana, van en aumento, a pesar de existir probablemente un subregistro en nuestro país, al no contar con un registro a nivel nacional que muestre la real magnitud de esta enfermedad". Por su parte, Leal A et al (2), indica que "los registros son en última instancia, el estándar de oro para la evaluación de los resultados de diversas intervenciones y los esfuerzos de prevención dirigidos a reducir la morbilidad y la mortalidad de uno de los más graves problemas de salud pública de nuestra época" y Curioso W (3), menciona "en muchos países, los sistemas de información en salud son débiles, incompletos y fragmentados. Sin embargo, existe un amplio consenso en la literatura para fortalecer los sistemas de información en salud en los países a nivel mundial”.

D'Agostino M (4), al referirse a las estrategias de salud en las Américas menciona: "Es posible revolucionar la práctica de la salud pública en las Américas a través de la implementación de herramientas basadas en web que permiten la extracción automatizada de grandes cantidades de datos masivos estructurados y no estructurados (datos, textos, tweets, mensajes, audios, videos) y que son generados por los comportamientos sociales. Este fenómeno es conocido en su sigla del idioma inglés como Big Data y forma parte de uno de los principales desafíos y oportunidades del presente y futuro cercano".

El registro de Cáncer de Lima Metropolitana en su última publicación (5), en sus páginas 19 y 20 presenta las fichas de incidencia y de mortalidad usada en su recolección manual, donde se consignan cerca de 27 variables necesarias para registrar solamente un caso de cáncer. Estas variables se pueden hallar en cuatro importantes fuentes de información electrónica, expresadas en la tabla 1.

El documento nacional de identificación (DNI) es el identificador universal que une a las cuatro fuentes de información electrónica, siendo el "Sistema de Información de la Red Nacional de Laboratorios de Salud Pública en el Perú (NETLAB)" (6), una herramienta en construcción que debe adaptarse y desarrollarse con el fin de ser un repositorio de todos los análisis de laboratorios, exámenes histológicos, citológicos, necropsias, así como también los informes de rayos $\mathrm{X}$, ecografías, tomografías, estudios de resonancia magnética, etc., incluyendo las variables del 22 al 26, pues estas son las que generan estadísticas descriptivas de monitoreo, mientras que las tres fuentes restantes, Registro Nacional de Identificación y Estado Civil (RENIEC), Sistema Informático Nacional de Defunciones (SINADEF) y el Sistema de Información en Salud (HIS-Health Information System) son

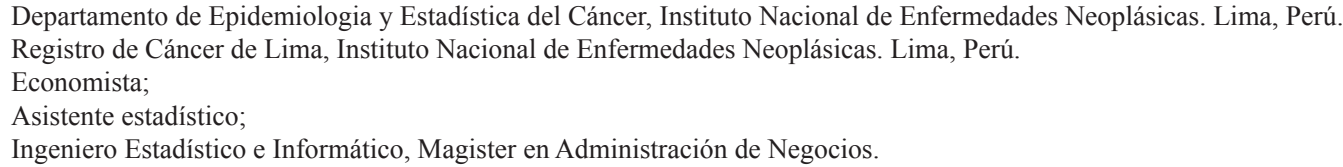


Tabla 1. Fuentes de Información Electrónica para un Registro Nacional de Cáncer Electrónico - RNC.

\begin{tabular}{|c|c|c|c|c|c|c|c|}
\hline & \multirow{2}{*}{$\begin{array}{l}\text { FUENTE RENIEC } \\
\text { DNI }\end{array}$} & \multicolumn{2}{|r|}{ FUENTE SINADEF } & \multicolumn{2}{|r|}{ FUENTE HIS } & \multicolumn{2}{|c|}{ FUENTE NETLAB } \\
\hline 1 & & 1 & DNI & 1 & DNI & 1 & DNI \\
\hline 2 & Primer Apellido & 2 & Primer Apellido & 2 & Primer Apellido & 2 & Primer Apellido \\
\hline 3 & Segundo Apellido & 3 & Segundo Apellido & 3 & Segundo Apellido & 3 & Segundo Apellido \\
\hline 4 & Nombres & 4 & Nombres & 4 & Nombres & 4 & Nombres \\
\hline 5 & Sexo & 5 & Sexo & 5 & Sexo & 5 & Sexo \\
\hline 6 & Estado Civil & 6 & Estado Civil & 9 & $\begin{array}{l}\text { Domicilio, Distrito de } \\
\text { Procedencia }\end{array}$ & 15 & $\begin{array}{l}\text { Cod. Hospital / } \\
\text { Establecimiento de } \\
\text { salud (EESS) }\end{array}$ \\
\hline 7 & Fecha Nacimiento & 8 & Lugar de Nacimiento & 15 & $\begin{array}{l}\text { Cod. Hospital / } \\
\text { Establecimiento de salud } \\
\text { (EESS) }\end{array}$ & 18 & Fecha de Examen \\
\hline 8 & Lugar de Nacimiento & 9 & $\begin{array}{l}\text { Domicilio, Distrito } \\
\text { de Procedencia }\end{array}$ & 16 & Fecha de Admisión & 19 & $\mathrm{~N}^{\mathrm{o}}$ de Examen \\
\hline 9 & $\begin{array}{l}\text { Domicilio, Distrito de } \\
\text { Procedencia }\end{array}$ & 10 & Fecha de Fallecido & 17 & $\mathrm{~N}^{\mathrm{o}}$ Historia Clínica & 20 & Muestra \\
\hline 10 & Fecha de Fallecido & 11 & Causas de Muerte & & & 21 & Diagnostico \\
\hline & & & $\begin{array}{l}\text { Distrito de } \\
\text { Fallecimiento }\end{array}$ & & & 22 & Base de Dx \\
\hline & & 13 & Sitio de Ocurrencia & & & 23 & Topografía \\
\hline & & 14 & Nombre de Médico & & & 24 & Morfología \\
\hline & & & & & & 25 & Diferenciación \\
\hline & & & & & & 26 & Estadio Clínico \\
\hline
\end{tabular}

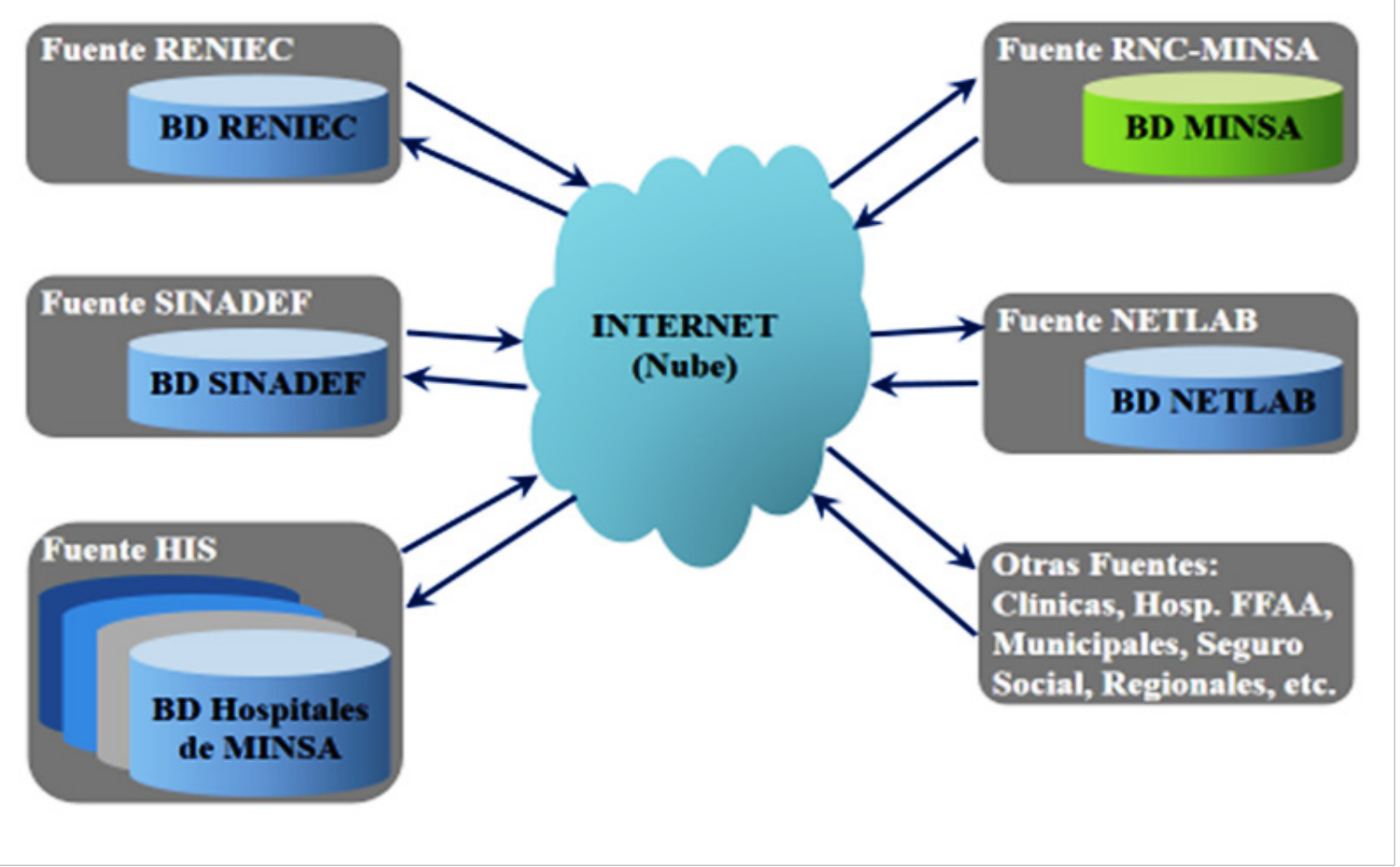

Figura 1. Operación Big Data para el registro nacional electrónico de cáncer (RNC). 
herramientas completamente desarrolladas a espera de una decisión política con el fin de unirse y compartir su información.

Los detalles técnicos de la plataforma de la base de datos (BD) para un Registro Nacional Electrónico de Cáncer (RNC) dependerán de varios factores, entre ellos el presupuesto asignado. En todo caso lo recomendable es usar un motor de base de datos MS SQL Server por la capacidad, confiabilidad y soporte del proveedor. Asimismo, la base de datos debe ir acompañada de una aplicación WEB mediante la cual se realizarían los registros, consultas, captura de datos de otras bases de datos, etc. La aplicación implicará un desarrollo ya sea interno o con un proveedor externo, el mismo que según su análisis y evaluación deberá recomendar un lenguaje y entorno de hardware y comunicación a usar. Se debe también considerar que la implementación de un sistema para RNC para administrar la información de la BD requerirá la definición de procesos y procedimientos, formatos, usuarios de diferentes perfiles, medidas de contingencia, etc. Todo ese trabajo lo realiza el equipo de desarrollo, un ingeniero de sistemas responsable y su equipo, para luego recién proceder con la programación, migración de data, pruebas, capacitación de usuarios y puesta en producción. En otras palabras, la creación e implementación y operación de la $\mathrm{BD}$ es sólo una etapa de un proyecto más grande que la incluye, que es precisamente un sistema de gestión de información para el RNC administrado por el Ministerio de Salud del Perú (MINSA). La operación Big Data se representa en la figura 1.

Finalmente, la propuesta de un sistema informático para el RNC está sujeto al desarrollo de NETLAB como un gran repositorio histórico de todos los exámenes que se haga el paciente en los diferentes sistemas de salud a nivel nacional, sea público o privado, identificado y registrado con su DNI, evitando la duplicidad de casos, el eficiente manejo de primarios múltiples, la obtención de informes cruzando variables y las estadísticas descriptivas de monitoreo, etc. Para lograr este objetivo es necesario la eficiente y planificada intervención política que puede acelerar metas y procesos ya que la parte técnica informática puede adaptarse.

\footnotetext{
Enrique Zevallos Santillan 1,a; 2,b,
} Luis Hurtado Málaga ${ }^{c}$

\section{Agradecimientos:}

Al Dr. Nelson Arias-Ortiz, por las opiniones brindadas para la redacción del presente artículo.

\section{Correspondencia:}

Enrique Zevallos Santillán

Correo electrónico: ezevallo@hotmail.com

\section{REFERENCIAS BIBLIOGRÁFICAS}

1. Pow-Sang M, Huaman M Retos para el diagnóstico precoz del cáncer de próstata en el Perú. Rev peru med exp salud pública. 2013; 30(1):124-128.

2. Leal A, Fernández-Garrote L Mohar-Betancourt A, Meneses-García A. The importance of registries in cancer control. Salud Pública Méx. 2016; 58(2): 309-316. (Citado el 26 de julio del 2016) Disponible en: http://www.scielo.org.mx/scielo.php?script=sci arttext\&pid=S0036-36342016000200026\&lng=es

3. Curioso W. eSalud en Perú: implementación de políticas para el fortalecimiento de sistemas de información en salud. Rev Panam Salud Publica. 2014; 35(5-6):437-441.

4. D'Agostino M. Estrategias de salud electrónica en la región de Las Américas: Situación actual y perspectivas. Rev peru med exp salud pública. 2015; 32(2):352-355. (Citado el 4 de enero del 2017) Disponible en http://www.scielo.org. pe/scielo.php?script $=$ sci_arttext\&pid=S172646342015000200021\&lng=es\&nrm=iso

5. Centro de Investigación en Cáncer Maes Heller, Instituto Nacional de Enfermedades Neoplásicas. Registro de Cáncer de Lima Metropolitana 20102012. Lima, Perú: Instituto de Enfermedades Neoplásicas; Setiembre 2016.

6. Vargas-Herrera J, Segovia-Juarez J, Garro Nuñez G. Sistema de información de la red nacional de laboratorios de salud pública en el Perú (Netlab). Rev Peru Med Exp Salud Pública. 2015; 32(2): 78-384. (Citado el 6 de enero del 2017) Disponible en: http://www.scielosp.org/scielo.php?script=sci arttext\&pid=S1726-46342015000200026\&lng $=$ en

Recibido: 31/08/2017 